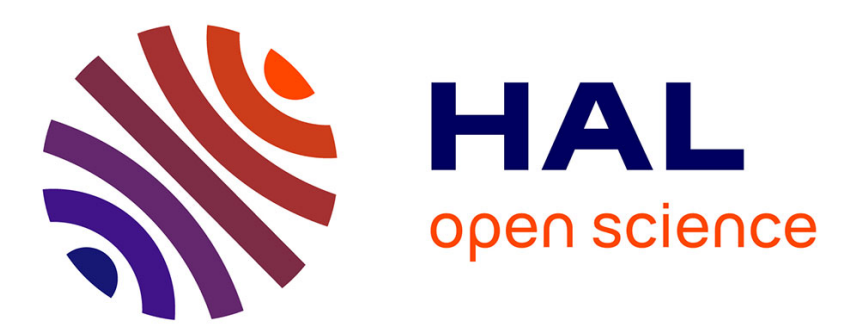

\title{
Inference of abrupt changes in noisy geochemical records using transdimensional changepoint models
}

Kerry Gallagher, Thomas Bodin, Malcolm Sambridge, Dominik Weiss, Malin Kylander, David Large

\section{To cite this version:}

Kerry Gallagher, Thomas Bodin, Malcolm Sambridge, Dominik Weiss, Malin Kylander, et al.. Inference of abrupt changes in noisy geochemical records using transdimensional changepoint models. Earth and Planetary Science Letters, 2011, 311, pp.182-194. 10.1016/j.epsl.2011.09.015 . insu-00641253

\section{HAL Id: insu-00641253 \\ https://hal-insu.archives-ouvertes.fr/insu-00641253}

Submitted on 15 Nov 2011

HAL is a multi-disciplinary open access archive for the deposit and dissemination of scientific research documents, whether they are published or not. The documents may come from teaching and research institutions in France or abroad, or from public or private research centers.
L'archive ouverte pluridisciplinaire HAL, est destinée au dépôt et à la diffusion de documents scientifiques de niveau recherche, publiés ou non, émanant des établissements d'enseignement et de recherche français ou étrangers, des laboratoires publics ou privés. 


\title{
Inference of abrupt changes in noisy geochemical records using transdimensional changepoint models
}

\author{
Kerry Gallagher ${ }^{1}$, Thomas Bodin ${ }^{2}$, Malcolm Sambridge ${ }^{2}$, Dominik Weiss ${ }^{3}$, \\ Malin Kylander ${ }^{4}$, David Large ${ }^{5}$
}

1 - Géosciences, Université de Rennes 1, Rennes, 35042, France

2 - Research School of Earth Sciences, Australian National University, Canberra, ACT 0200, Australia

3 - Dept. of Earth Science and Engineering, Imperial College London, London SW7 2AZ, England

4 - Dept. of Geological Sciences, Stockholm University, 10691 Stockholm, Sweden

5 - Dept of Chemical and Environmental Engineering, University of Nottingham, University Park, Nottingham, NG7 2RD, England

Corresponding author :

Kerry Gallagher, kerry.gallagher@univ-rennes1.fr, +33 (0)2 23236081 


\begin{abstract}
1 Abstract
We present a method to quantify abrupt changes (or changepoints) in data series, represented as a function of depth or time. These changes are often the result of climatic or environmental variations and can be manifested in multiple datasets as different responses, but all datasets can have the same changepoint locations/timings. The method we present uses transdimensional Markov chain Monte Carlo to infer probability distributions on the number and locations (in depth or time) of changepoints, the mean values between changepoints and, if required, the noise variance associated with each dataset being considered. This latter point is important as we generally will have limited information on the noise, such as estimates only of measurement uncertainty, and in most cases it is not practical to make repeat sampling/measurement to assess other contributions to the variation in the data. We describe the main features of the approach (and describe the mathematical formulation in supplementary material), and demonstrate its validity using synthetic datasets, with known changepoint structure (number and locations of changepoints) and distribution of noise variance for each dataset. We show that when using multiple data, we expect to achieve better resolution of the changepoint structure than when we use each dataset individually. This is conditional on the validity of the assumption of common changepoints between different datasets. We then apply the method to two sets of real geochemical data, both from peat cores, taken from NE Australia and eastern Tibet. Under the assumption that changes occur at the same time for all datasets, we recover solutions consistent with those previously inferred qualitatively from independent data and interpretations. However, our approach provides a quantitative estimate of the relative probability of the inferred changepoints, allowing an objective assessment of the significance of each change.
\end{abstract}

Keywords : Transdimensional changepoint models, Geochemical data, Bayesian modelling, climate change 


\section{Introduction}

32 A major issue in the interpretation of geochemical data (represented as depth or time series) is the detection of changes in trends. Reliable identification of short-term variations superimposed on long-term trends is critical for answering questions about uniformity in

the rates of geological processes. The research effort towards understanding climate change often focusses on the inference of rapid or abrupt changes in the mean signal over time. Such environmental changes are recorded by geochemical proxies (e.g. McDermott 2004, Carslaw et al. 2006, Kylander et al. 2007, 2009, 2010, Gutjahr et al 2008, Cloy et al. 2008, Yasahuru et al. 2008, Ruggieri et al 2009, Large et al 2009, Cole et al. 2009, Palmer et al. 2010, Burton et al. 2007, 2010). Ideally, recognition of signals from data should based on sound qualitative interpretation, but also involve quantitative inference from the observations, allowing for possibly unknown noise levels in the data.

Here we recognise that the definition of abrupt or rapid is subjective. The definition and identification of change also depends on the form of the trend we expect between changes. We define abrupt changes as statisically significant variation in the trend over a scale of one or two samples of the total dataset. Furthermore, we note that the conversion of depth to time (or age) generally involves calibration of a depth-age relationship, which itself will have uncertainty (e.g. Thompson and Goldstein 2006, Kylander et al. 2009, 2010). For data collected from one borehole for example, these uncertainties will not change the positions of the underlying changes, only affecting the inference of absolute timing, but may become important for the inference of simultaneous changes in data from different locations.

To provide a brief overview of some approaches for inferring abrupt changes in geochemical records, we draw on a selection of published work, including some of our own. Large et al. (2009) described geochemical data including C, N, H concentrations and 
$\mathrm{C}, \mathrm{H}$ and $\mathrm{O}$ isotopes from a $6 \mathrm{~m}$ core taken in the Hongyuan peatland, eastern Qinghai-

57 Tibetan Plateau. The core material was dated back to 9.6 kiloyears using ${ }^{14} \mathrm{C}$. The interpreted palaeoenvironmental history was linked to the climate variations in northwest

Pacific, the El Niño-Southern Oscillation, movement of the Intertropical Convergence Zone and the East Asian Monsoon. This interpretation was based on a qualitative visual inspection and comparison to other proxy data and interpretations.

Ruggieri et al. (2009) developed a method to infer Milankovitch-type cycles from geochemical $\left(\delta^{18} \mathrm{O}\right)$ data,. They allow for discrete changes (changepoints) between which the trend (defined by superimposed sine functions) can change abruptly. They apply their model to 2 sets of benthic $\delta^{18} \mathrm{O}$ isotope data with time ranges going back to 2500 and 5000 kiloyears. As these authors state, an important limitation of their method is that they do not include the number of change points as a parameter to be inferred directly. Instead they examine the variation of the data fit as a function of the number of changepoints, and try to identify the upper limit such that adding more changepoints makes little difference to the data fit. One additional limitation of this approach is that the inference will depend on the errors inherent in the data, although the data fit function adopted by Ruggieri et al (2009) does not incorporate a data error term explicitly. In general, we expect a more complex model (i.e. more changepoints) given more precise data. Often however, we do not have reliable estimates of the data errors and then choosing a suitable model becomes an issue.

Tomé and Miranda (2004), looking for changes in linear trends, fit gradients to time series, subject to constraints on the minimum distance between changepoints, and the magnitude of the changes in trends. The approach requires a user to specify a range for number of changepoints, and finds best fitting functions for each value of the number of change points in turn. Although the authors state that they can examine a series of the sums of squares of residuals for each model (with different numbers of changepoints) to choose 
81 an appropriate value, they do not explicity do this. Rather they seem to favour visual inspection to select a preferred model, which is required to have a constant distance between the changepoints.

Finally, Kylander et al. (2007) analysed rare earth elements and lead isotopes from samples in an Australian peatland as inorganic proxies for climatic variations, reflected in atmospheric dust. The changepoint modelling approach described in Denison et al. (2002) was adapted to allow common changepoints for multiple datasets. Kylander et al. (2007) used Eu abundance and $\mathrm{Pb}$ isotopes to infer the changepoints, under the assumption that trends in the data between the changepoints could be expressed as a simple linear regression function (a constant value or constant slope). In contrast to previous approaches, the number of changepoints was a parameter to be inferred directly. Moreover, the problem is formulated in a Bayesian framework, and provides probability distributions on the number of changepoints and on the changepoint locations.

In this paper, we present a general approach to infer an unknown number of changepoints when the errors, or the noise variances, are unknown for one or more datasets. The data can be irregularly spaced in depth (or time) and there is no requirement for different datasets to be sampled at the same depths (time). We specify nothing about the spacing between changepoints, and the approach we present generalises to any linear function between changepoints. A key difference in the approach we present here to that of 100 Denison et al. (2002) is that we decouple the estimates of the data noise variance and the 101 model parameters. A related approach to deal with poorly constrained errors was described by Malinverno and Briggs (2004) applied to seismic traveltime inversion for 1D velocity structure (and see also Malinverno and Parker, 2006). 
106 although most of the mathematical details are given in the supplementary material. The 107 method we present includes not only estimation of the number and location of 108 changepoints, the regression functions between changepoints, but also the distribution of 109 the data noise variance (if it is unknown or considered unrepresentative). We conclude with 110 some examples of the application of the method to synthetic and real data and a brief 111 discussion/summary.

\section{Changepoint modelling and noise}

The general problem can be stated as follows (and see figure 1) ; given one or more sets of (geochemical) data, $f\left(x_{i}\right), i=1, N$, at positions $x_{i}$, representing a depth or time series, with either known or unknown levels of noise, can we identify the underlying trends or signal (e.g. the mean or a more general regression function) and the locations of discrete changes in the trends ? Often ,we may want to infer the same changepoint locations, but with different signals in each dataset.

In general we do not know how many changes are appropriate and ideally we should estimate this from the data. The problem becomes a transdimensional inverse problem (that is we do not specify in advance the number of unknown parameters, e.g.

124 Sambridge et al. 2006). Furthermore, there is a trade-off between the level of noise in the 125 data, and how well we expect to fit the data. Here we broadly follow the philosophy of 126 Scales and Snieder (1999) in considering noise as that part of the data that we do not 127 wish/expect the model to explain. The spread in a geochemical dataset can then be divided 128 into the variation $\left(\sigma_{G P}^{2}\right)$ due to time/depth varying geological processes (which we are 129 interested in understanding) and the variation due to geological $\left(\sigma_{G N}^{2}\right)$ and analytical $\left(\sigma_{A N}^{2}\right)$ noise. Here geological noise might arise from spatial or temporal variations in small scale 
131 or short term processes, local geological/biological/atmospheric variability and analytical

132 noise (or more commonly, errors) typically can arise from factors such as instrumental drift

133 or calibration from imperfect standards. In general we can express the total variance, $\sigma_{T}^{2}$ as

134

135

136

137

138

139

140

144

148

149

150

151

152

153

When we are interested in identifying trends and changes in trends, we would like to choose a model that adequately explains the variation due to geological process, and then the resiudals between the model predictions and the observed data reflectsthe 2 sources of noise as defined above. We want good control on the noise (or noise variance) as this will directly influence how well we should fit our data. Intuitively, we can see that if we consider a series of scattered data with lower noise variance than is appropriate then we will tend to fit many changepoints. An extreme case would be if we assume no noise, then we will fit the data perfectly (with a changepoint between each data point). On the other hand if we regard the data as more noisy than they really are, then we will tend to fit a model that has too few changepoints.

\subsection{Bayesian formulation of the change-point modelling problem}

Underpinning the Bayesian approach is that unknowns are expressed in terms of probability density functions (e.g. Tarantola and Valette 1982). A common form of Bayes' rule is

$$
p(m \mid d) \alpha p(d \mid m) p(m)
$$

where $p(m \mid d)$ is the probability density function (PDF) of the unknown model parameter vector, $m$, containing the unknowns, given the data vector, $d ; p(d \mid m)$ is the likelihood 
154 function which is effectively the probability of the data, $d$, being observed given the model, $m$. The likelihood increases as the model fits the data better relative to the data noise and the form of the likelihood depends on the statistical character of the noise on the data. Finally, $p(m)$ is the prior PDF on the model (that is what we think we know about $m$ before we have the data). The aim of Bayesian inference is to try and estimate the posterior PDF, $p(m \mid d)$ as this characterises all we need to know about the distribution of model parameter values, given the prior and the information contained in the data (incorporated through the likelihood). Useful references for Bayesian inference are by Box and Tiao (1973), Lee (1989), Bernardo and Smith 1994) and Gelman et al (2004).

\subsubsection{Model parameters}

In a changepoint problem, the unknown model parameters are the number of changepoints $(n)$, their locations $(c)$, the parameters of a regression function between the changepoints $(A)$ and the noise level $(\sigma)$ for each dataset being considered. Thus we can write a general model vector, $\boldsymbol{m}$, as

$$
m=(n, c, A, \sigma)
$$

in which $c, A$, and $\sigma$ can all be vectors.

We write the unknown locations of changepoints as $\mathrm{c}_{\mathrm{i}}, \mathrm{i}=1, n$ (note that $n$ itself is also an unknown). We refer to the region between each changepoint as a partition, and a predictive regression function, $f_{i}(x)$, is defined whose parameters $(A)$ depend on the data in that partition. Thus $f_{i}(\mathrm{x})$ refers to the regression function within the partition at the left of change point $c_{i}$ (if these are considered on a horizontal axis). As there are $\mathrm{n}+1$ partitions for $\mathrm{n}$ change points, we define $c_{0}$ as the location of the first data point so the regression function $\mathrm{f}_{1}$ in the first partition is defined between $c_{0}$ and $c_{1}$. 
179 as depth (or time) and observed data respectively, the linear regression function between 180 changepoints can be written as

$$
f(x)=\sum_{i=1}^{M} \alpha_{i} G_{i}(x)
$$

182 where $\boldsymbol{G}_{i}$ represents a specified basis function and $\alpha_{i}$ represents an unknown coefficient, 183 which can be thought of as weights on each basis function. For example, the common 184 straight line relationship given by $d_{\text {pred }}=\alpha_{1}+\alpha_{2} x$, is written as a vector-matrix equation,

$$
d_{\text {pred }}=G^{t} A
$$

186 where the superscript $t$ represents the matrix transpose, and

187

$$
G=\left(\begin{array}{cc}
1 & x_{1} \\
\cdot & \cdot \\
1 & x_{i} \\
\cdot & \cdot \\
1 & x_{k}
\end{array}\right), \quad A=\left(\begin{array}{l}
\alpha_{1} \\
\alpha_{2}
\end{array}\right)
$$

188

189 A model with a constant value (normally the mean) is written as

190

$$
G=\left(\begin{array}{c}
1 \\
\cdot \\
1 \\
\cdot \\
1
\end{array}\right), \quad A=\alpha_{1}
$$

191 As we can separate the basis functions from the coefficients, this is a linear problem (linear 192 in terms of the unknown coefficients). Here, we us only constant value regression functions 193 between changepoints. However the approach we present generalises to any linear function, 194 allowing for more gradual transitions between states (e.g. linear drift).

\subsubsection{Data likelihood}




$$
p\left(d_{1}, d_{2} \ldots d_{N_{d}} \mid m\right)=\prod_{l=1}^{N_{d}} \prod_{i=1}^{n} \prod_{j=1}^{k_{i}} \frac{1}{\left(2 \pi \sigma_{l}^{2}\right)^{\frac{1}{2}}} e^{-\frac{1}{2}\left(\frac{d_{i, j, l}-f_{i . l}(x)}{\sigma_{l}}\right)^{2}}
$$

and the variance of this noise distribution may be poorly (or not) known. This is equivalent to the residuals between the observations and the predictions being normally distributed with mean of zero and a variance of $\sigma^{2}$. If we have reliable and representative estimates of the noise variances, we can use the values in the likelihood instead of $\sigma$. In practice, the noise term determines the uncertainty in fitting the data which, due to geological complexity, is usually greater than the reported analytical precision. Given this assumption, a Gaussian likelihood function for observed data (using $d=d_{o b s}$ ) in the i-th partition, given the predictions with a particular set of model parameters $(\boldsymbol{m})$ is

$$
p\left(d_{i} \mid m\right)=\prod_{j=1}^{k_{i}} \frac{1}{\left(2 \pi \sigma^{2}\right)^{\frac{1}{2}}} e^{-\frac{1}{2}\left(\frac{d_{i, j}-f_{i}(x)}{\sigma}\right)^{2}}
$$

where the subscript $j$ refers to the $k_{i}$ data in the i-th partition (i.e. the region bounded by changepoints $c_{i}$ and $c_{i-1}$, with $c_{0}$ defined the lowest value of the data locations) and $d_{i, j}$ is the j-th observation in partition i.

More generally, for n partitions and $N_{d}$ different datasets, we have the joint likelihood function, 
219 Given we subsequently assume a constant value in a partition, an appropriate form to chose 220 values for the predictive function $(f)$ is a normal distribution centred on the mean value 221 (and a variance equal to the variance) of the data in that partition. In this case, values for 222 the regression model parameters (A) can be drawn from this distribution while the mean is 223 the most probable value from the posterior distribution in a Bayesian formulation, 224 equivalent to the maximum likelihood estimate. We could use a similar approach for any 225 linear function with unknown coefficients, using standard least squares inverse methods 226 (e.g. Menke 1989) to find the maximum likelihood values and the covariance matrix for the 227 coefficients for a given partition, and draw samples for the parameters.

\subsubsection{Prior distributions}

230 In a Bayesian formulation, we need to specify prior distributions on all unknown 231 parameters. The priors reflect what we consider reasonable to assume about the possible 232 values for each parameter. Bayes's rule lets us use the information about the model 233 parameters contained in the data to update our prior information (i.e. to produce the 234 posterior distribution). If the posterior distribution is the same as the prior, then the data 235 have told us nothing we did not already know.

$$
\begin{aligned}
p(m) & =p(n) p(c, A, \sigma \mid n) \\
& =p(n) p(c \mid n) \prod_{l}^{N_{d}} p\left(A_{l} \mid c, n\right) p\left(\sigma_{l} \mid c, n\right)
\end{aligned}
$$

239 where $\mathrm{N}_{\mathrm{d}}$ is the number of datasets. The choice of priors in our formulation is

240 straightforward (usually uniform between a specified minimum and maximum value) and 241 are given in Supplementary material, SM2. 


\section{Markov chain Monte Carlo method for solving the changepoint problem}

244 In using the Bayesian formulation described above, our goal is to generate a collection, or 245 ensemble, of values approximating the posterior distribution, whose form we do not know 246 in advance. As we also do not know the number of changepoints, the problem becomes 247 what is known as transdimensional, where the number of model parameters itself becomes 248 an unknown. To solve this problem we use a generalised version of Markov chain Monte 249 Carlo (MCMC) sampling, known as Reversible Jump MCMC (Green 1995, 2003). A 250 general introduction to MCMC methods is given by Gilks et al. (1996), a review of 251 transdimensional Markov chains is given by Sisson (2005) and Gallagher et al. (2009) 252 present an overview of the general methodology and its application to Earth Science 253 problems. Specific applications to Earth Science problems have been presented by 254 Malinverno (2002), Malinverno and Leaney (2005), Stephenson et al (2006), Jasra et al. 255 (2006), Sambridge et al. (2006), Hopcroft et al. (2009), Charvin et al. (2008), Bodin and 256 Sambridge (2009) and Piana Agostinetti and Malinverno (2010). We give brief overview of 257 MCMC in the Supplementary material, SM1, while here we describe the aspects important 258 for the transdimensional changepoint problem. The mathematical details for the MCMC 259 implementation we adopt for the changepoint problem are given in Supplementary 260 material, SM2 and SM3.

MCMC is an iterative method, and at each iteration, we consider 2 sets of model 262 parameters, the current and proposed models $\left(\boldsymbol{m}_{\boldsymbol{c}}\right.$ and $\left.\boldsymbol{m}_{\boldsymbol{p}}\right)$. The procedure for a given 263 iteration can be described as follows

264 (i) Randomly perturb the current model to produce the proposed model 265 (ii) Randomly accept or reject the proposed model (in terms of replacing the 266 current model), according to the acceptance criterion ratio (see equation A1.2 in Supplementary material, SM1) . 
268 In principle, after many iterations, the MCMC sampler should converge to a stable 269 configuration (that is sampling according to the posterior distribution) and the final stage of

270 MCMC is to use the sampling to infer characteristics and uncertainties for the model.

272 (i) Model perturbations/moves

273 As stated above, the sampling should converge to the target posterior distribution. 274 However, the efficiency of the method does depend on choosing a reasonable proposal 275 function to avoid moving too slowly around the model space as of result of the 276 perturbations being either too small or too big (see figure 2 of Gallagher et al. 2009). The 277 scale of model parameter perturbations can be tuned to achieve a reasonable balance 278 between accepting and rejecting proposed models.

279 For the changepoint problem, we define 5 types of model perturbation or move :

$280 \quad$ 1. Change the location of a changepoint

2812 . Change the regression function (mean estimate) in a partition between 2 changepoints

3. Change the value of noise for a dataset (if appropriate for that dataset)

4. Add a new changepoint (birth)

5. Remove an existing changepoint (death)

287 Each type of move has specified probability of being selected (which forms the jump 288 proposal, $R$, referred to in Supplementary Material), and these probabilities need to sum to 289 unity. In our problem, these are set to $0.2,0.15,0.15,0.25$ and 0.25 in the order listed 290 above. The birth and death probabilities need to be modified to avoid having more/less 291 changepoints than the maximum/minimum values $\left(\mathrm{n}_{\max } / \mathrm{n}_{\min }\right)$. We do this by setting the 292 birth (death) probability to 0.5 (0.0) for a model with $\mathrm{n}_{\min }$ changepoints, and the death 
293 (birth) probability to $0.5(0.0)$ for a model with $\mathrm{n}_{\max }$ changepoints. Having selected a 294 perturbation type for a particular iteration, all other parameters are kept constant.

\section{6 (ii) Acceptance criterion}

For the purposes of describing how we accept or reject the proposed model, we use

298 a simplified form of acceptance criterion ratio appropriate for 2 models with the same number of model parameters (and the full expressions for the transdimensional case are given in the Supplementary material). This can be written as a ratio of probabilites, given as

$$
\alpha\left(m_{p}, m_{c}\right)=\operatorname{Min}\left[1, \frac{\left.p\left(m_{p}\right) p\left(d \mid m_{p}\right)\right)\left(m_{c} \mid m_{p}\right)}{\left.p\left(m_{c}\right) p\left(d \mid m_{c}\right)\right)\left(m_{p} \mid m_{c}\right)}\right]
$$

where Min[1,Z] means we take the minimum of 1 and $\mathrm{Z}$. The terms $p(m)$ and $p(m \mid d)$ are the prior and likelihood probabilities for a particular model (and so define the posterior probability, at least up to the constant of proportionality). We have already introduced the concepts of the prior and likelihood functions (and where appropriate, the problem specific details are given in Supplemntary material, 2).

The proposal probability, $q\left(m_{p} \mid m_{c}\right)$ determines how we move from the current model to proposed model (step (i) above). The theory underlying MCMC requires us to be able to reverse such a move (so we need to also include the reverse proposal probability in the ratio). If we consider prior distributions to be uniform (i.e. all models have the same

311 probability), and if we have proposal distributions that are symmetric, then we can write equation 14 above as

$$
\alpha\left(m_{p}, m_{c}\right)=\operatorname{Min}\left[1, \frac{p\left(d \mid m_{p}\right)}{p\left(d \mid m_{c}\right)}\right]
$$

314 This is just a ratio of the likelihoods (i.e. the probability of the proposed and current models 315 producing the observed data). Thus if the proposed model fits the observed data better than 
the current model (so it has a higher likelihood), then the likelihood ratio is $>1$, and then

318 model, the ratio is $<1$, and then $\alpha($.$) is set to \mathrm{p}\left(\boldsymbol{d} \mid \boldsymbol{m}_{\boldsymbol{p}}\right) / \mathrm{p}\left(\boldsymbol{d} \mid \boldsymbol{m}_{\boldsymbol{c}}\right)$, which itself is always $>0$.

319 The final step in an iteration requires us to generate a uniform random number, $\mathrm{u}$, between

3200 and 1 , and compare this to $\alpha($.$) . If \mathrm{u}<=\alpha($.$) we accept the proposed model (and this$

321 becomes the current model for the next iteration), otherwise we reject it (and we retain the 322 current model for the next iteration). From this, we can see that (given the assumptions 323 about flat priors and symmetrical proposal functions) we will always accept a proposed 324 model fits the data better as $\mathrm{u}$ is always $<=1$. If we consider a proposed model that fits the 325 data almost as well as the current model (say $\alpha()=$.0.95 ), then, on average, we will accept 326 the proposed model $95 \%$ of the time. For a proposed model considerably worse than the current model in terms of data fit (say $\alpha()=$.0.05 ), then, on average, we will only accept 328 the proposed model $5 \%$ of the time. If the posterior distribution resembles a normal 329 distribution, we can see that this process will tend to concentrate the sampling under and around the peak (the higher probability region), but also allows us to sample less good models (out in the tails of the distribution). In fact, the number of accepted samples for each model is proportion to the posterior probability of that model and the ensemble of accepted models then is a good approximation of the posterior distribution.

For the first 3 moves described in (i) above, the number of model parameters is constant and the acceptance ratio is given by equation 11 . For the birth and death moves, the dimensions of the current and proposed model are different and it is necessary to use 338 the acceptance ratio given in Supplementary Material 1 (equation A1.2). Intuitively, we 339 might expect that models with more parameters will tend to provide a better fit to the observed data, and then that the sampler would tend always to increase the number of 
model parameters towards the maximum. However, to demonstrate how MCMC operates during transdimensional moves, we can consider again a simplified form of the acceptance criterion as

$$
\alpha\left(m_{p}, m_{c}\right)=\operatorname{Min}\left[1, \frac{p\left(m_{p}\right) p\left(d \mid m_{p}\right)}{p\left(m_{c}\right) p\left(d \mid m_{c}\right)}\right]
$$

If all the parameters are independent and we consider a proposed model with 1 more parameter more than the current model (all other parameters being the same), then, with the prior on the extra parameter given as $\mathrm{p}\left(\mathrm{m}_{\mathrm{p}, \mathrm{n}+1}\right)$ we can write this as

$$
\begin{aligned}
\alpha\left(m_{p}, m_{c}\right) & =\operatorname{Min}\left[1, \frac{p\left(m_{p,+1}\right) p\left(m_{c}\right) p\left(d \mid m_{p}\right)}{p\left(m_{c}\right) p\left(d \mid m_{c}\right)}\right] \\
& =\operatorname{Min}\left[1, \frac{p\left(m_{p,+1}\right) p\left(d \mid m_{p}\right)}{p\left(d \mid m_{c}\right)}\right]
\end{aligned}
$$

351 If both models fit the observations equally well (the likelihoods have the same value), then as $\mathrm{p}\left(\mathrm{m}_{\mathrm{p}, \mathrm{n}+1}\right)<1$, we have

$$
\alpha\left(m_{p}, m_{c}\right)=p\left(m_{p,+1}\right)
$$

Thus, in this special case, the acceptance probability is equal to prior probability on the extra model parameter. In other words, even when the fit to the observations is as good as the current model, the proposed model (with more parameters) is less likely to be accepted, by a factor equal to the prior probability of the extra parameter.

More specifically, when we propose an increase in the number of changepoints

360 (birth), an increase in likelihood function will tend to encourage acceptance of the proposed 361 model. However the decrease in the prior ratio will tend to discourage acceptance due to 
the increased dimensionality of the space. Overall, the algorithm always prefers a large partition rather than two small partitions with similar mean values (which would have similar likelihood values). This is an example of a property of Bayesian inference referred to as 'natural parsimony', which means that given a choice between a simple and complex models that provide similarfits to data, the simpler one will be favoured (e.g. Jeffreys and Burger 1992, Mackay 1992, Bretthorst 1993, O' Ruanaidh and Fitzgerald 1996, Sivia 1996, Jaynes 2003).

(iii) Calculation of model and uncertainties

Typically the MCMC sampling is run for many $\left(10^{4}-10^{6}\right)$ iterations, and includes an initial period during which the samples are not yet from the target posterior distribution. This is known as burn-in and these samples are discarded before making inference from the posterior distribution. Gilks et al. (1996) show examples of these characteristic behaviours as a guide for their recognition and we discuss this later with the examples. The post-burnin samples should then provide a good approximation to the posterior distribution for the model parameters, i.e. $p(\boldsymbol{m} \mid \boldsymbol{d})$. This can be visualised for one model parameter by plotting a histogram. We can also calculate the expected model as a average, i.e.

which is effectively a weighted mean, in which the weighting is the posterior probability for each model. Similarly the variance and co-variance of model parameters are given by standard formulae. Finally, we can readily calculate the 95\% credible intervals by ordering the samples for a particular variable, and simply identifying the upper and lower $2.5 \%$ of the distribution as the $95 \%$ credible interval. 
Rather than choosing the best data fitting model, which tends to be overly complex, our preferred final solution is given by the expected model (equation 16) with 95\% credible intervals around the regression function parameters, and the distributions on the number and locations of changepoints. When a large number of models are added together, their

391 partitions overlap so the average model is continous and smooth. An advantage of this is that we can produce a model that contains the features common to the majority of sampled models, but also can be more complex (yet smoother) than any individually sampled model.

\section{Examples of changepoint modelling}

We first use synthetic data to demonstrate that we can recover the known signal and noise terms. In this example, we discuss how to assess whether the MCMC sampler has performed adequately. Subsequently, we apply the method to 2 sets of real geochemical et al. 2009).

\subsection{Synthetic data}

404 The synthetic data are shown in figures 2a,b,c. We randomly selected 4 changepoints and 405 different mean value functions in each partition to produce 150 irregularly distributed 406 samples for each dataset and added noise with different levels to each. We used these data, 407 assuming unknown noise variance, to infer the distributions on the number and locations of 408 changepoints and the noise variance.

As stated earlier, we tune the proposal functions to achieve adequate sampling of 410 the model parameters. Among the more common ways to assess if these input parameters 411 are appropriate are to examine the rate of acceptance (typically around 30\%, although 10- 
$60 \%$ may be adequate, e.g. Brooks et al., 2003), and also the behaviour of the likelihood or model parameters as a function of iteration (they should show no long term trend, and

414 ideally resemble white noise). However, for birth and death we can not readily control the 415 acceptance rates, which can be much lower $(<5 \%)$ in this problem.

416 We choose a proposal function scale ( $\theta$ in equation A2.6) that is proportional to the 417 range (maximum and minimum values) of particular parameter. We use 0.2, 0.05 and 0.025 418 of the prior ranges for the regression function (here the mean), the changepoint locations 419 and the noise, respectively. We make exploratory runs in which we monitor the acceptance 420 rates over $10^{4}-10^{5}$ iterations, and adjust the scaling parameters accordingly. In this problem, 421 if the acceptance rate is too high, the scaling parameters are too small, and vice-versa. 422 Having tuned the proposal functions, we run the chain for $5 \times 10^{5}$ iterations, with a burn-in 423 of $2.5 \times 10^{5}$ iterations.

In figure 3, we show the log likelihood (data fit), the number of partitions and the 425 sampling for the 3 noise parameters. During the early part of sampling (figure 3a,b), there 426 is clearly structure in the chain. The initial log-likelihood is about $-1.2 \times 10^{4}$, but even over 427 the initial 5000 iterations we see that the sampler quickly arrives in better regions of the 428 model space, and the log-likelihood increases rapidly, even though the number of 429 changepoints is decreasing. Over the same sampling period, the noise values have not 430 equilibrated either, the blocky structure, indicative of relatively poor movement around the 431 model space (or mixing). In contrast, the post-burn parts of the chain show that the 432 sampling appears to have reached equilibrium. There are no significant trends in the 433 sampling (they look like white noise), and the number of partitions is sampled between 4 434 and 7.

435 As described in section 3, we use the post-burn-in samples to calculate the expected 436 (or average) changepoint structure, the 95\% credible intervals around it, the probability of 
having changepoints over the range of the samples and also the mean and distribution on 438 the noise values for each dataset. In figures 2d,e,f we show the 3 datasets, with the mean 439 estimated noise value as error bars, together with the expected changepoint structure, and in 440 figure 4 we show the distributions on the noise parameters. It is clear, by comparison with 441 figure 2, the changepoint structure has been recovered well, with no spurious features, and 442 also the mean noise is in good agreement with the original values. In figure 5 we show the 443 distribution on the inferred number of changepoints, demonstrating that the inference leads 444 to about $80 \%$ probability there are 4 . This is conditional on all the model assumptions (a 445 finite number of discrete changes with constant mean values in each partition), although 446 these are appropriate in this example.

To demonstrate the influence of different datasets, we ran each dataset 448 independently, using the same parameters as the joint run and the results are shown in 449 figure 6. Again the main changepoint structure is recoved, although we see that some small 450 scale artifacts have been introduced for individual datasets. This leads to a slightly different 451 distribution on the number of changepoints, although all 3 datasets still have 4 as the most 452 probable, with the frequency of 5 changepoints, relative to 4, being higher than in the joint 453 model (25\% compared with 98\% dataset 1 , 65\% datset 2 , and 30\% dataset 3 ). In practice, it 454 is unlikely that we would be primarily interested in the absolute number of changepoints, 455 but rather in where changes are inferred to occur. If we have multiple datasets, and expect 456 them to have the same changepoint structure, then we recommend modelling them together 457 for consistency in the changepoint structure. 


\subsection{Real data examples}

In the real data examples described below, we follow a similar approach as described above. We monitored the acceptance rate on all variable parameters, and where relevant we adjusted the proposal function scales to achieve an acceptance rate of 20-40\%.

\subsubsection{Lynch’s Crater, Australia}

The first real data example we consider is from Kylander et al (2007), who undertook geochemical analyses on a $13 \mathrm{~m}$ section equivalent to $~ 50 \mathrm{Kyr}$ from a peatbog at Lynch’s crater, north-east Australia. They reported $\mathrm{Pb}$ isotope and Rare Earth Element data, used as proxies for climate change, and in particular for variations in air-transported mineral dust sources. Here we follow that paper, and consider the ${ }^{206} \mathrm{~Pb} /{ }^{207} \mathrm{~Pb}$ isotope ratios and the Europium anomaly, $\left(\mathrm{Eu} / \mathrm{Eu}^{*}\right)_{\mathrm{PAAS}}$, which is a measure of $\mathrm{Eu}^{2+}$ fractionation from $\mathrm{Eu}^{3+}$ relative to the adjacent ions, $\mathrm{Sm}^{3+}$ and $\mathrm{Gd}^{3+}$ ( $\mathrm{Eu}^{*}$ being the geometric mean of these two).

We first use this example to demonstrate the inference of changepoints either representing the noise with the analytical errors or estimating the noise variance directly from data. In terms of analytical errors, those for the $\mathrm{Pb}$ isotope ratio were determined from a long-term series (nearly 2 years) measurements of the NBS 981 standard. In the absence of an equivalent estimate from repeat measurements, we assumed $10 \%$ of the observed value for the Eu anomaly. The mean noise variances on $\mathrm{Pb}$ and the $\mathrm{Eu}$ anomaly are $3.86 \times 10^{-4}$ and 0.123 , respectively.

In figure 7 we show the inferred changepoint structure using these specified errors in the data likelihood, and also the case in which we also infer the noise variances, for each dataset in terms of a probability distribution (figure 8). Clearly, the structure in the first case is dominated by the $\mathrm{Pb}$ isotope ratios (which have relatively small analytical errors). The mean number of changepoints is $106( \pm 4,1 \sigma)$ and it is difficult to make meaningful 
sense of these results. In the second case, where we estimate the noise variance, the mean number of changepoints is $6( \pm 1,1 \sigma)$, and correspondingly, the mean of the noise variances on the $\mathrm{Pb}$ isotope data and the $\mathrm{Eu}$ anomaly are 0.00913 and 0.0341 , respectively. In this case, the inferred noise variance on the Eu anomaly is about 3-4 times smaller than the assumed $10 \%$, while for the $\mathrm{Pb}$ isotopes, it is about 24 times larger than the analytical level. In terms of the inferred changepoint locations, the major peaks around 150 and $620 \mathrm{~m}$ correspond to the two most significant changes inferred by Kylander et al. (2007). The shallowest changepoint is related to a change to warm, wet conditions while the second is 492 change from humid to arid. The $3^{\text {rd }}$ peak around $820 \mathrm{~m}$ in our results also corresponds to a 493 lesser change inferred by the earlier work. We refer the reader to Kylander et al. (2007) and 494 Muller et al. (2008), for a more detailed discussion of the environmental significance of 495 these changes.

We also ran the 2 datasets individually, assuming the errors were unknown, and the 497 results were very similar in terms of the error distributions. The Pb isotope data however 498 only required one significant changepoint (around 820m), while the Eu anomaly data 499 produced essentially the same result as the joint modeling.

\subsubsection{Hongyuan, Eastern Tibet}

502 Large et al. (2009) presented a series of geochemical and physical property measurements 503 from a $6 \mathrm{~m}$ deep section, equivalent to $10 \mathrm{Kyrs}$, of the Hongyuan peatbog in eastern Tibet. 504 The aim of this study was to assess the relative influences of the Indian and east Asian 505 monsoons, and to relate this to other inferences of climate variations in China. Here, we use 506 the $\mathrm{C}, \mathrm{N}, \mathrm{H}$ and $\delta^{13} \mathrm{C}$ analyses, together with the bulk density and carbon density to make 507 quantitative inference of changepoints. In this case, we have no specific information 508 concerning the errors for each dataset, so we also need to infer the noise variance. 
510 noise levels are lower than the standard deviation of each dataset ( 2-3 times lower), except

511 for the $\delta^{13} \mathrm{C}$ dataset, for which the inferred noise variance is similar in magnitude to the 512 variation in data. The summary diagram of Large et al. (2009) (their figure 7) compares 513 their data to previous studies, and in particular of inferred periods of cold, dry (permafrost) 514 periods relative to warmer, wetter periods. Thus our inferred changepoints should 515 correspond to times when these conditions switch. Apart from the relatively low amplitude 516 probability changepoint inferred around $200 \mathrm{~cm}$ and the recent variations $(<50 \mathrm{~cm}$, 517 attributed to disturbance as a consequence of Yak grazing by Large et al, 2009), the 518 changepoints agree well with those inferred by a qualitative comparison of regional 519 datasets from China by Large et al. (2009) (see figure 9).

520 Although we do not show the results here, we also ran these 6 datasets individually.

521 As we might now expect, the details of the changepoint location structure differs between 522 each dataset. Also, the mean values of the estimated noise levels were lower (by between 52310 and 60\%) than for the joint model. This latter result is also not unexpected as the joint 524 modelling tends to compromise (increase) the noise variance to accommodate common 525 changepoints for multiple datasets. While there is clearly common information, it is not 526 easy to identify reliably the changepoints by considering these datasets individually. Again 527 we recommend joint modelling of multiple datasets if we anticipate a common changepoint 528 structure for a particular problem.

\section{Summary}

531 Changepoints can be defined as abrupt changes in trends (such as the mean, gradient or any 532 function) over depth or time. In this paper, we have presented a new approach to 533 changepoint modelling, applicable to multiple datasets with common changepoint 
534 locations, allowing for unknown noise variance in each dataset. The approach is based on 535 Bayesian transdimensional Markov chain Monte Carlo and we estimate the changepoint 536 structure in terms of distributions for number and location of changepoints, the regression 537 function parameters and the noise variance on multiple datasets. Here, we have considered 538 the regression function in terms of a constant value between 2 changepoints, but the 539 approach generalises to any linear function of the data. In any transdimensional problem, 540 the solution (i.e. the number and location of changepoints) is strongly influenced by the 541 assumed noise variance. Our approach, in allowing us to estimate the noise variance 542 directly from the data, is particularly useful when we do not have reliable estimates of the 543 data error/noise, or perhaps only consider analytical errors (i.e. we neglect natural variation 544 due to geological complexity) and so implicitly assume the data are more precise than is 545 perhaps advisable. Furthermore, the Bayesian approach we adopt is naturally parsimonious 546 and avoids inferring unwarranted complexity when finding the changepoint structure. Thus 547 we expect to favour models with fewer changepoints, while still achieving an adequate fit 548 to the observed data.

549 Using synthetic data, we have demonstrated that we can recover the changepoint 550 structure and the noise variances reliably. When dealing with multiple datasets, we assume 551 that all datasets contain the same changepoint locations, but the response, or regression 552 functions, and noise variances are different. The approach we present can be generalised 553 readily to allow for different noise variance between partitions, if required. Additionally, 554 the different datasets can be irregularly spaced in depth (or time) and there is no need for 555 the data to be sampled at the same depths (time). The details of the solutions will depend on 556 which datasets are used (i.e. singly or jointly) and we recommend using joint modelling if 557 the assumption of common changepoints is considered valid. This assumption is perhaps 558 best assessed from the understanding of geochemical behaviour in different enviromental 
559 systems. Certainly, the results are more coherent and generally easier to interpret than by 560 combining results from individual dataset modelling. Applications of the method to real 561 datasets from NE Australia and eastern Tibet provide results in agreement with previous 562 qualitative interpretations based on visual inspection. However, our approach is preferable 563 as it is more objective, explicity incoporates the noise variance (either known or unknown), 564 allows us to assess quantitatively the relative importance of the inferred changepoint 565 structure, and we obtain probability distributions on all parameters. Finally, directions for 566 future work would be to consider transdimensional regression functions (for example we 567 estimate the order of a polynomial which could be different between partitions) and to 568 allow for uncertainty in depth to age conversions (which will be important when comparing 569 records from different locations). 


\section{Acknowledgements}

We thank the French Australian Science and Technology (FAST) program for support during this work. We thank 2 anonymous referees for comments on an earlier version. 


\section{REFERENCES}

Bernardo, J. \& Smith, A.F.M., 1994. Bayesian Theory, John Wiley and Sons, Ltd., Chichester.

Bodin, T. \& Sambridge, M., 2009. Seismic tomography with the reversible jump algorithm, Geophys. J. Int., 178, 1411-1436, doi:10.1111/j.1365-246X.2009.04226.x

Box, G.E.P. and Tiao, G.C. 1973. Bayesian Inference in Statistical Inference, Wiley \& Sons, New York, pp 588.

Bretthorst, G. L. (1993). An introduction to model selection using probability theory as logic. In Heidbreder, G., editor, Maximum Entropy and Bayesian Methods, pages 142. Kluwer Academic, Dordrecht.

Brooks, S.P., Giudici, P., \& Roberts, G.O., 2003. Efficient construction of reversible jump Markov chain Monte Carlo proposal distribution, J. R. Stat. Soc., Ser. B90 J. Stat. Method. 65, 3-39.

Burton, G.R., Rosman, K.J.R., Candelone, J.P., Burn, L.J., Boutron, C.F. \& Hong, S.M., 2007. The impact of climatic conditions on $\mathrm{Pb}$ and $\mathrm{Sr}$ isotopic ratios found in Greenland ice, 7-150 ky BP. Earth Planet. Sci. Lett. 259, 557-566.

Burton, K.W., Gannoun, A. \& Parkinson, I.J., 2010. Climate driven glacial-interglacial variations in the osmium isotope composition of seawater recorded by planktic foraminifera. Earth Planet. Sci. Lett. 295 58-68

Carslaw, D.C., Ropkins, K \& M.C. Bell., 2006. Change-Point Detection of Gaseous and Particulate Traffic-Related Pollutants at a Roadside Location. Env. Sci. Tech. 40, 22. 6912-6918.

Charvin, K., Gallagher, K., Hampson, G. \& Labourdette, R., 2009. A Bayesian approach to infer environmental parameters from stratigraphic data 1: Methodology, Basin Research, 21, 5-25.

Cloy, J.M., Farmer, J.G., Graham, M.C., MacKenzie, A.B. \& Cook, G.T., 2008. Historical records of atmospheric $\mathrm{Pb}$ deposition in four Scottish ombrotrophic bogs: an isotopic comparison with other records from western Europe and Greenland. Glob. Biogeochem. Cy. 22, GB2016.

Cole, J.M., Goldstein, S.L., deMenocal, P.B.. Hemming, S. R., \& Grousset, F.E., 2009, Contrasting compositions of Saharan dust in the eastern Atlantic Ocean during the last deglaciation and African Humid Period. Earth Planet. Sci. Lett., 278, 257-266

Denison, D.G.T., Holmes, C.C., Mallick, B.K. \& Smith, A.F.M., 2002. Bayesian Methods for Nonlinear Classification and Regression, Wiley, Chichester, 2002.

Gallagher, K., Charvin, K., Nielsen, S., Sambridge, M. and Stephenson, J., 2009. Markov chain Monte Carlo (MCMC) sampling methods to determine optimal models, model resolution and model choice for Earth Science problems, J. Mar. Petrol. Geol., 26, 525-535.

Gelman, A., Carlin, J.B., Stern, H.S. and Rubin, D.B. 2004. Bayesian Data Analysis. Texts in Statistical Science, Chapman \& Hall. Pp. 668

Gilks, W.R., Richardson, S. \& Spiegalhalter, D.J., 1996. Markov Chain Monte Carlo in Practice. London: Chapman \& Hall. 
Green, P.J., 1995. Reversible jump Markov chain Monte Carlo computation and Bayesian model determination. Biometrika, 82, 711-732.

Green, P.J., 2003. Transdimensional MCMC, in: P.J. Green, N. Hjort and S. Richardson (Eds.), Highly Structured Stochastic Systems, Oxford Statistical Sciences Series, Ch.6, pp. 179-196.

Gutjahr, M., Frank, M., Stirling, C.H., Keigwin, L. \& Halliday, A.N., 2008. Tracing the Nd isotope evolution of North Atlantic Deep and Intermediate waters in the western North Atlantic since the Last Glacial Maximum from Blake Ridge sediments. Earth Planet. Sci. Lett. 266, 61-77.

Hopcroft, P., Gallagher, K. \& Pain, C.C., 2009. A Bayesian Partition Modelling approach to resolve spatial variability in climate records from borehole temperature inversion, Geophys. J. Int, 178, 651-666, doi: 10.1111/j.1365-246X.2009.04192.

Jasra, A. Stephens, D.A., Gallagher, K. \& Holmes. C.C., 2006. Analysis of geochronological data with measurement error using Bayesian mixtures Math. Geol., 38, 269-300.

Jaynes, E. T. (2003). Probability Theory: The Logic of Science. Cambridge University Press, Cambridge, U. K.

Jefferys, W. H. and Berger, J. O. (1992). Ockham's razor and Bayesian analysis. American Scientist, 80:64-72.

Kylander, M.E., Muller, J., Weust, R., Gallagher, K., Garcia-Sanchez, R., Coles, B. J. \& Weiss, D.J., 2007. Rare earth element and $\mathrm{Pb}$ isotope variations in a $52 \mathrm{kyr}$ peat core from Lynch's Crater (NE Queensland, Australia): Proxy development and application to paleoclimate in the Southern Hemisphere, Geochim. Cosmochim. Acta, 71, 942960.

Kylander, M.E., Weiss, D.J. \& Kober, B., 2009. Two high resolution terrestrial records of atmospheric $\mathrm{Pb}$ deposition from New Brunswick, Canada, and Loch Laxford, , Scotland. Sci Tot Environ 407, 1644-1657.

Kylander, M.E., Klaminder J., Bindler R., \& Weiss D.J. 2010. Natural lead isotope variations in the atmosphere. Earth Planet. Sci. Lett., 290, 44-53.

Large, D.J., Spiro, B., Ferrat, M., Shopland, M., Kylander, M. Gallagher, K., Li, X., Chengde, S., Shen, C., Possnert, G., Zhang, G., Darling, W. \& Weiss, D. 2009. The influence of climate, hydrology, and permafrost on Holocene peat accumulation at 3500m on the Eastern Qinghqi-Tibetan Plateau, Quat. Sci. Rev. 28, 3303-3314.

Lee, P.M., 1989. Bayesian Statistics : An Introduction, Edward Arnold, pp 294.

MacKay, D. J. C. 1992. Bayesian interpolation. Neural Comp., 4, 415-447.

Malinverno, A. 2002. Parsimonious Bayesian Markov chain Monte Carlo inversion in a nonlinear geophysical problem. Geophys. J. Int., 151, 675-688.

Malinverno, A. \& Briggs, V., 2004. Expanded uncertainty quantification in inverse problems : Heirachical Bayes and empirical Bayes, Geophysics, 69, 1005-1016.

Malinverno, A. and Leaney, W. S. 2005. Monte Carlo Bayesian look-ahead inversion of walkaway vertical seismic profiles. Geophys. Prosp., 53, 689-703.

Malinverno, A. \& Parker, R.L, 2006.Two ways to quantify uncertainty in geophysical inverse problems, Geophysics, 71, W15.

McDermott, F., 2004. Palaeo-climate reconstruction from stable isotope variations in 
speleothems: a review, Quat. Sci. Rev. 23 901-918

Menke, W., 1989. Geophysical Data Analysis : Discrete Inverse Theory Revised Edition, International Geophysics Series 45, Academic Press, pp 289.

Muller, J., M. Kylander, R.A.J. Wüst, D. Weiss, A. Martinez-Cortizas, A.N. LeGrande, T. Jennerjahn, H. Behling, W.T. Anderson, and G. Jacobson, 2008. Possible evidence for wet Heinrich phases in tropical NE Australia: The Lynch's Crater deposit. Quaternary Sci. Rev., 27, 468-475, doi:10.1016/j.quascirev.2007.11.006.

O' Ruanaidh, J. J. K. and Fitzgerald, W. J. (1996). Numerical Bayesian Methods Applied to Signal Processing. Springer-Verlag, New York.

Palmer M.R., Brummer, G.J, Cooper, M.J., Elderfield, H., Greaves, M.J., Reichart, G.J., Schouten, S. \& Yu, J.M., 2010. Multi-proxy reconstruction of surface water pCO2 in the northern Arabian Sea since 29 ka. Earth Planet. Sci. Letts 295 49-57

Piana Agostinetti, N. \& Malinverno A., 2010. Receiver function inversion by transdimensional Monte Carlo sampling, Geophys. J. Int. 181, 858-872.

Ruggieri, E. Herbet, T., Lawrence, K.T. \& Lawrence, C.E., 2009. Change point method for detercting regime shifts in paleoclimatic time series : Application to $\delta^{18} \mathrm{O}$ time series of the Plio-Pleistocene, Palaeocean., 24, PA1204, doi:10.1029/2007/PA001568

Sambridge, M., Gallagher, K., Jackson, A. \& Rickwood, P., 2006. Transdimensional inverse problems, Model Comparison and the Evidence. Geophys. J. Int.l,167, 528542.

Scales, J., \& Snieder, R., 1998. What is noise?, Geophysics, 63, 1122-1124.

Sisson, S.A., 2005, Transdimensional Markov Chains: A Decade of Progress and Future Perspectives, J. Am. Stat. Assoc., 100, 1077-1090.

Sivia, D. S. (1996). Data Analysis: A Bayesian Tutorial. Clarendon Press, Oxford.

Stephenson, J., Gallagher, K. \& Holmes, C., 2006. Low temperature thermochronology and modelling strategies for multiple samples 2 : Partition modelling for 2D and 3D distributions with discontinuities, Earth Plan. Sci Letts, 241, 557-570.

Tarantola, A. \& Valette, B., 1982. Inverse problems = quest for information, J. Geophys., $50,159-170$.

Thompson, W.G., \& Goldstein, S.L., 2006. A radiometric calibration of the SPECMAP timescale, Quat. Sci. Rev. 25, 3207-3215.

Tomé, A.R. and Miranda, P.M.A. 2004. Piecewise linear fitting and trend changing points of climate parameters, Geophys. Res. Letts. 31, L02207, doi :10.1029/2003GL019100.

Yasuhara, M. Cronin, T.M., deMenocal, P.B., Okahashi, H. \& Linsley, B.K. 2008, Abrupt climate change and collapse of deep-sea ecosystems. Proc. Nat. Acad. Sciences., 105, 1556-1560. 


\section{Figure Captions}

Figure 1. An example of the changepoint problem. We have a set of noisy data (dots), with a common noise variance (the noise is Gaussian, and $1 \sigma$ value is shown as the error bar in the bottom right). The underlying function from which the data were generated is shown by the solid line. The function is discontinuous, with 4 changepoints at $x=2,5,6$, and 8 . In a real problem, the model parameters are the number of changepoints $(n)$, the locations of the changepoints $\left(\mathrm{x}_{\mathrm{i}}, \mathrm{i}=1, n\right)$ and the values of the function in each region (in this case, this is just the mean value of the data between each changepoint).

\section{Figure 2}

(a,b,c). The 3 synthetic datasets (grey dots), with 4 common changepoints. The noise scale ( $\sigma$ from equation 7 ) is given in the top left, with an error bar $\pm 1 \sigma$ shown just below. The true regression function for each dataset is shown by the solid line.

(d,e,f) Changepoint structure inferred for the 3 synthetic dataset. The solid line is the inferred function (relative to the lefthand axis), and the lighter dashed lines represent the 95\% credible intervals on this function. The continuous lines represent the probability of a changepoint (relative to the right hand axis). The error bars are drawn using the mean value of the noise variances for each data set (see figure 4).

Figure 3.

(a) Log-likelihood (LL) and the number of changepoints (n) for the initial 5000 iterations

(b) The sampled values for the 3 noise terms over the initial 5000 iterations.

(c) As (a), but during the post-burn-in sampling

(d) As (b), but during the post-burn-in sampling 
Figure 4. Inferred distributions of the noise variances for each data set. The true values are shown as the heavy vertical lines.

Figure 5. Inferred distribution on the number of changepoints for the data in figure 3.

Figure 6. As figure 2,d,e,f,, but each data set was modelled individually.

Figure 7. Inferred changepoint model for the data from Kylander et al. (2007). Their inferred climatic-related varitations are indicated by the grey bar bars at the base of each graph.

(a) $\mathrm{Pb}$ isotope data using the analytical errors for the noise term.

(b) Eu anomaly data, using $10 \%$ of the observed value as the noise term

(c) As (a), but we infer the noise variance (mean value shown as the error bars)

(d) As (b), but we infer the noise variance (mean value shown as the error bars)

Figure 8. Inferred distributions of the noise variance for the two datasets of Kylander et al (2007).

Figure 9. Inferred changepoint model for the data from Large et al. (2009). Their inferred climatic-related variations are indicated by the grey bar bars at the base of each graph.

(a) H, (b) N, (c) C, (d) Total Carbon, (e) $\delta^{13} \mathrm{C}$, (f) density

Figure 10. Inferred distributions of the noise variance for the 6 datasets of Large et al (2009). The sequence of graphs is the same as in figure 9. 